Revista PSICOLOGIA, 2015, Vol. 29 (1), 11-22

\title{
"Entre marido e mulher, a crise mete a colher": A relação entre pressão económica, conflito e satisfação conjugal
}

\author{
Sara I. Ferreira ${ }^{1}$, Marta F. Pedro ${ }^{1}$ e Rita Francisco ${ }^{1}$ \\ ${ }^{1}$ Faculdade de Psicologia, Universidade de Lisboa
}

\begin{abstract}
Resumo: Considerando a crise económica que Portugal atravessa, e com base no modelo de stress familiar económico (MSFE), o presente estudo teve como objectivo investigar o papel mediador do stress emocional na relação entre a percepção de pressão económica e o conflito e satisfação conjugais. Procurou-se, ainda, alargar a aplicabilidade do MSFE, testando-o no contexto português, bem como contribuir para um maior conhecimento do modelo através da utilização de uma abordagem de análise baseada no actor-partner interdependence model. Participaram no estudo 208 casais portugueses, casados ou em união de facto, com filhos adolescentes. Os resultados mostraram uma relação indirecta entre a percepção de pressão económica e o conflito e satisfação conjugais, mediada através do stress emocional de cada cônjuge. Os resultados indicaram também a existência uma relação directa entre a percepção de pressão económica e o conflito conjugal de ambos os elementos do casal, bem como entre a percepção de pressão económica e a satisfação conjugal dos homens, divergindo do preconizado pelo MSFE e sugerindo possíveis influências culturais.
\end{abstract}

Palavras-chave: Crise económica; Pressão económica; Stress emocional; Conflito conjugal; Satisfação conjugal; Modelo de stress familiar económico.

Economic crisis and marital quality: The relationship between economic pressure and marital conflict and satisfaction: Considering the economic crisis currently affecting Portugal, and based in the family stress model of economic pressure (FSM), the present study aims to investigate the mediating role of emotional stress in the relationship between the perception of economic pressure, and marital conflict and satisfaction. It was also sought to expand the FSM's applicability, testing it in the Portuguese context, using an actor-partner interdependence model approach. Participants were 208 Portuguese couples, married or living together, with adolescent children. The results showed an indirect pathway between the perception of economic pressure and marital conflict and satisfaction, mediated by both spouses emotional stress. The results also showed direct effects between the perception of economic pressure and women and men's marital conflict, as well as direct effects between the perception of economic pressure and men's marital satisfaction, suggesting possible cultural influences.

Keywords: Economic crisis; Economic pressure; Marital conflict; Marital satisfaction; Family stress model of economic pressure.

As dificuldades económicas são um dos principais factores de conflito conjugal (e.g., Papp, Cummings, \& Goeke-Morey, 2009), conduzindo a sentimentos de preocupação constante relativamente a despesas domésticas e de apoio familiar (Kinnunen \& Feldt, 2004). O modelo de stress familiar económico, desenvolvido por Conger e Elder (1994), foi um dos primeiros a investigar o impacto da situação económica na relação conjugal, sugerindo que a percepção de dificuldades financeiras prejudica a qualidade da mesma, originando instabilidade na interacção entre os cônjuges. Apesar de a investigação demonstrar, de forma consistente, o impacto das dificuldades financeiras no conflito (e.g., Aytaç \& Rankin, 2009; Papp et al., 2009; Robila \& Krishnakumar, 2005) e na satisfação conjugal (e.g., Dakin \& Wampler, 2008; Kwon, Rueter, Koh, \& Ok, 2003), a maioria dos estudos tem utilizado constructos agregados das respostas de ambos os elementos do casal (Ponnet, Wouters, Goedemé, \& Mortelmans, 2013), impossibilitando a análise das influências mútuas e interdependências entre os cônjuges. Por outro lado, apesar da crise financeira que atravessa Portugal e das preocupações económicas serem uma presença constante nas famílias, até à data não há estudos publicados sobre a influência da percepção de pressão económica nos casais portugueses.

Dados de contacto para correspondência: Marta Pedro, Faculdade de Psicologia da Universidade de Lisboa, Alameda da Universidade, 1649-013, Lisboa, Portugal.E-mail: mmpedro@psicologia.ulisboa.pt 


\section{Contexto Económico em Portugal}

A sociedade actual atravessa um período de recessão económica mundial, levando a que muitas famílias enfrentem problemas financeiros significativos (Brinkman, Pee, Sanogo, Subran, \& Bloem, 2010). A crise económica afecta Portugal desde 2007, tendo sido implementado um Programa de Assistência Económica e Financeira (PAEF) em 2011.

De acordo com o Instituto Nacional de Estatística (INE, 2013), em 2012 a taxa de desemprego rondava os $15 \%$ e, em $2013,27.4 \%$ da população vivia em risco de pobreza e/ou exclusão social. Como resposta a esta realidade, muitas famílias viram-se obrigadas a cortar despesas em diversas áreas, tais como alimentação, saúde, educação, entre outras (SEDES, 2012). Em 2013, a Associação Portuguesa para a Defesa do Consumidor (DECO) recebeu mais de 29 mil pedidos de ajuda por parte de famílias carenciadas, um aumento de $26 \%$ relativamente ao ano anterior. 0 desemprego (32\% das famílias) e os cortes salariais (30.6\%) foram as principais causas apontadas para os pedidos de apoio efectuados (sobretudo relacionados com sobre-endividamento). Até Setembro de 2012, 38\% de famílias portuguesas admitiu chegar ao final do mês com 300 euros negativos na conta bancária e cerca de 70 mil agregados familiares assumiram não ter dinheiro suficiente para uma alimentação adequada (DECO, 2013). Embora Portugal tenha saído oficialmente do PAEF em 2013, sem necessidade de recorrer a um programa cautelar, existe ainda um longo percurso até à recuperação económica e social do país.

Estes dados salientam a necessidade de investigar a relação entre as dificuldades financeiras que afectam as famílias portuguesas, o stress individual e a qualidade da relação conjugal (nomeadamente, ao nível do conflito e da satisfação conjugal).

\section{Modelo de Stress Familiar Económico}

O modelo de stress familiar económico (MSFE), desenvolvido por Conger e colaboradores (Conger \& Elder, 1994; Conger \& Donnellan, 2007; Conger, Conger, \& Martin, 2010) postula que a existência de dificuldades financeiras, tais como rendimentos baixos ou acontecimentos financeiros negativos (e.g., perder o emprego), origina pressão económica nas famílias, afectando o funcionamento familiar e individual. Esta pressão económica corresponde à avaliação subjectiva que o indivíduo faz das suas circunstâncias financeiras, sendo frequentemente considerada mais importante para o estudo do seu funcionamento do que as condições objectivas de problemas financeiros (Conger et al., 1999; Robila \& Krishnakumar, 2005). Constituindo um indicador da resposta das famílias à sua situação financeira, a pressão económica refere-se à experiência psicológica associada à falta de capacidade para fazer face às despesas (Conger \& Elder, 1994; Mistry, Lowe, Benner, \& Chien, 2008), incluindo: (a) necessidades materiais insatisfeitas, tais como alimentação ou vestuário, (b) incapacidade para pagar contas ou fazer face às despesas, e (c) necessidade de efectuar cortes em despesas básicas (e.g., cuidados de saúde) (Conger \& Donnellan, 2007). De acordo com o MSFE, a experiência deste tipo de pressões e exigências confere significado psicológico às dificuldades financeiras. Assim, este modelo preconiza que a existência de elevados níveis de pressão económica provoca stress emocional em ambos os elementos do casal (e.g., depressão, ansiedade), conduzindo a um aumento do conflito conjugal e diminuindo a satisfação conjugal (Conger et al., 1990). O MSFE tem sido testado com recurso a várias metodologias e investigado em diversas populações, como E.U.A. (e.g., Conger et al., 2002), Canadá (Davis \& Mantler, 2004), Europa (e.g., Falconier \& Epstein, 2011; Ponnet et al., 2013), Turquia (Aytaç \& Rankin, 2009) e Coreia (Kwon et al., 2003).

\section{o Papel Mediador do Stress Emocional}

A investigação tem vindo a demonstrar o impacto da percepção de pressão económica no bem-estar e saúde mental dos indivíduos, associando-a a stress emocional, nomeadamente: sintomas de depressão, ansiedade, hostilidade e dificuldades sociais (e.g., Conger et al., 2002; Falconier \& Epstein, 2011; Kwon et al., 2003; Mistry et al., 2008; Wickrama et al., 2010). Na Grécia, por exemplo, a reduzida capacidade para satisfazer necessidades básicas, aliado ao aumento de dívidas resultante da forte crise económica que afecta o país, provocaram o aumento de casos de depressão (Economou, Madianos, Peppou, \& Patelakis, 2012).

Por outro lado, vários estudos indicam que a percepção de pressão económica afecta indirectamente o conflito e a estabilidade conjugal, através do stress emocional provocado nos elementos do casal (e.g., Conger et al., 1999; Conger et al., 2002; Cutrona et al., 2003; Kinnunen \& Feldt, 2004; Robila e Krishnakumar, 2005). Conger e colaboradores (1992) constataram que a elevada percepção de pressão económica se relacionava indirectamente com o conflito conjugal através do aumento dos níveis de depressão e de hostilidade, diminuindo as interacções carinhosas e de apoio na díade conjugal. Mais recentemente, um estudo realizado na Bélgica confirmou que, quando os casais vivenciam stress e 
preocupação decorrentes da situação económica actual e/ou futura, há um aumento de sintomas depressivos e, consequentemente, do conflito conjugal (Ponnet et al., 2013).

Menos investigado tem sido o impacto da percepção de pressão económica na satisfação conjugal. Num dos poucos estudos que consideraram a satisfação conjugal no contexto de dificuldades financeiras, os casais recém-casados capazes de pagar dívidas experienciavam níveis mais elevados de satisfação conjugal, contrariamente aos casais cujas dívidas se mantinham ou aumentavam, os quais sofriam um declínio da satisfação conjugal (Dew, 2008). A investigação tem-se centrado maioritariamente no conflito entre os elementos do casal, descurando as especificidades da satisfação conjugal ao abordá-la numa perspectiva simplista, de mera ausência de conflito e dificuldades conjugais (e.g., Fincham, Stanley, \& Beach, 2007). Pretende-se assim contribuir para preencher esta lacuna na literatura, investigando a associação entre a percepção de pressão económica e o conflito e satisfação entre os cônjuges, considerando a importância da satisfação conjugal no bem-estar dos indivíduos (e.g., Fincham et al., 2007).

Embora grande parte das evidências empíricas demonstrem uma relação indirecta entre a percepção de pressão económica e a satisfação e conflito conjugal, estudos recentes sugerem também a existência de uma relação directa, nomeadamente, em populações afro-americanas (e.g., Cutrona et al., 2003) e países fora dos EUA, como a Coreia (Kwon et al., 2003), Turquia (Aytaç \& Rankin, 2009) ou Finlândia (Kinunnen \& Feldt, 2004). Estes resultados poderão estar relacionados com diferentes tradições culturais e/ou económicas de sociedades fora dos EUA, as quais não são ainda totalmente compreendidas (Conger et al., 2010). Mais ainda, estas evidências contraditórias reforçam a necessidade de aplicação do MSFE em diferentes contextos culturais, como aliás tem sido realizado por alguns autores (e.g., Leinonen, Solantaus, \& Punamäki, 2002; Robila \& Krishnakumar, 2005).

O contexto português, em particular, apresenta especificidades que poderão contribuir para uma maior compreensão do funcionamento do MSFE. Nomeadamente, Portugal tem sido considerado por vários autores como um caso único no contexto europeu (e.g., Aboim, 2010a), sobretudo no que diz respeito a dois aspectos: a elevada participação das mulheres no mercado de trabalho (60\% acima da média da União Europeia e a mais elevada dos países do sul da Europa) e a persistente diferenciação dos papéis de género dentro do sistema familiar (Aboim, 2010b; Amâncio, 2007). Portugal constitui ainda um exemplo de uma sociedade onde coexistem atitudes de género contraditórias. Se, por um lado, se valoriza uma participação equilibrada entre homens e mulheres no mercado de trabalho, em que ambos contribuem para o rendimento familiar, por outro, na esfera familiar, defende-se ainda uma visão tradicional do papel de género, na qual a mulher é a principal responsável pelo desempenho das tarefas da vida privada, como a prestação de cuidados aos filhos e o trabalho doméstico (Aboim, 2010b). Deste modo, apesar da elevada taxa de empregabilidade feminina, em Portugal ainda predomina o modelo tradicional do homem provedor, responsável pelo "ganha-pão" da família. Estes factores, em conjunto com os resultados contraditórios quanto à natureza da relação (directa ou indirecta) entre a percepção de pressão económica e o conflito e satisfação conjugais, reforçam a pertinência de se investigar o MSFE no contexto português, considerando que as especificidades culturais da sociedade portuguesa poderão contribuir para uma maior compreensão deste modelo.

\section{Especificidades de Género na Relação entre Pressão Financeira, Satisfação e Conflito Conjugal}

Há ainda evidências empíricas que sugerem possíveis diferenças de género ao nível do impacto das dificuldades financeiras no funcionamento conjugal. Nomeadamente, alguns estudos indicam que a relação entre dificuldades financeiras e a satisfação e o conflito conjugal é mais pronunciada nos homens, mostrando que o seu comportamento está mais associado a problemas financeiros que o comportamento das mulheres (e.g., Conger et al., 1990; Liker \& Elder, 1983). Aniol e Snyder (1997), por exemplo, constataram que as preocupações financeiras estavam associadas à satisfação conjugal dos homens, mas não à satisfação conjugal das mulheres. A maioria dos estudos tem investigado como é que o stress experienciado por um indivíduo num domínio da sua vida (e.g. o contexto económico) pode originar stress ou dificuldades noutro domínio (e.g. a relação conjugal). Este processo, designado de spillover, corresponde assim à transferência intra-individual de afectos e comportamentos entre contextos diferentes (e.g., Bolger, DeLongis, Kessler, \& Wethington, 1989; Larson \& Almeida, 1999). Contudo, a transferência inter-individual de afecto e comportamentos de um elemento do casal para o outro, denominado de crossover, tem sido consideravelmente menos investigado no contexto de pressão económica. Os estudos acerca dos processos de crossover na relação conjugal têm indicado que este poderá ser unidirecional, mostrando que o stress dos homens tem impacto no bem-estar das mulheres (e.g., Jones \& Fletcher, 1993; Pittman, Solheim, \& Blanchard, 1996; Westman, Etzion, \& Danon, 2001). Estes dados sugerem que as mulheres poderão ser mais susceptíveis a processos de crossover enquanto receptoras do stress dos homens (e.g., Larson \& Almeida, 1999). Contudo, a investigação em países onde 
os papéis de género são relativamente igualitários e, como tal, os assuntos financeiros desempenham igual importância na vida dos homens e das mulheres, tem salientado a natureza bidirecional dos processos de crossover, ou seja, ocorrendo de homens para mulheres e de mulheres para homens (e.g., para uma revisão ver Westman, 2001). Outros estudos não encontraram diferenças ao nível do género (e.g., Gudmunson, Beutler, Israelsen, McCoy, \& Hill, 2007). As inconsistências dos dados acima descritos salientam a necessidade de se continuar a investigar as diferenças entre os cônjuges e a influência mútua entre ambos, no contexto de percepção de pressão económica.

Importa ainda referir que a literatura sobre o impacto da pressão económica na relação conjugal tem prestado pouca atenção às influências mútuas existentes entre os elementos do casal, sendo poucos os estudos que seguem uma abordagem diádica dos dados (e.g., Kinnunen \& Feldt, 2004; Ponnet et al., 2013). A maioria da investigação nesta área (e.g., Conger et al., 1999; Wickrama et al., 2010) tem isolado as variáveis reportadas por homens e mulheres, e/ou utilizado constructos agregados das respostas de ambos os elementos do casal, abordagem que aliás tem sido criticada por ignorar a interdependência inerente às relações de casal. 0 presente estudo pretende contribuir para esta lacuna na literatura, investigando a influência mútua entre os elementos do casal com base no Actor-Partner Interdependence Model (APIM; Kenny, Kashy, \& Cook, 2006). O APIM é uma abordagem que permite examinar o impacto das variáveis preditoras de um indivíduo nas suas variáveis consequência (actor effects), assim como nas variáveis consequência do cônjuge (partner effects). No APIM, os actor effects são indicativos de spillover, enquanto que os partner effects são referentes a crossover. O APIM representa assim a natureza diádica das relações de casal, postulando que os membros de uma díade se influenciam mutuamente na forma de partner effects, estabelecendo um interdependência entre ambos.

\section{Objectivos e hipóteses}

Considerando o contexto de crise financeira que caracteriza Portugal actualmente, o presente trabalho apresenta três objectivos principais: (1) replicar o MSFE de Conger e colaboradores (Conger \& Elder, 1994; Conger et al., 2010) numa amostra portuguesa, procurando expandir a aplicabilidade do modelo num contexto cultural diferente; (2) testar o papel mediador do stress emocional dos elementos do casal, na relação entre percepção de pressão económica e conflito e satisfação conjugal; e (3) investigar a influência mútua entre os elementos do casal com recurso a uma análise dos dados baseada no ActorPartner Interdependence Model (APIM; Kenny, Kashy, \& Cook, 2006).

Tendo em conta o MSFE e os resultados dos estudos empíricos apresentados, são consideradas as seguintes hipóteses:

Hipótese 1: a percepção de pressão económica estará directamente relacionada com o conflito e com a satisfação conjugal;

Hipótese 2: o stress emocional dos homens e das mulheres estará directamente relacionado com o conflito e com a satisfação conjugal dos cônjuges;

Hipótese 3: o stress emocional de ambos os elementos do casal irá mediar a relação entre percepção de pressão económica e conflito e satisfação conjugal. Assim, a percepção de pressão económica estará positivamente associada ao stress emocional de ambos os elementos do casal (H3a) e o stress emocional de ambos os elementos do casal estará positivamente associado ao conflito conjugal e negativamente associado à satisfação conjugal do próprio (actor effects) e do cônjuge (partner effects) (H3b).

\section{MÉTODO \\ Participantes}

A amostra foi constituída por 416 participantes (208 casais heterossexuais), casados ou em união de facto, residentes na zona da Grande Lisboa, com filhos entre os 12 e os 21 anos de idade $(M=15.02, D P=$ 1.89). Os homens têm idades compreendidas entre os 30 e os 78 anos $(M=46.58, D P=6.49)$ e as mulheres entre os 29 e os 58 anos $(M=46.63, D P=5.08)$. Apresentam diferentes níveis de escolaridade, sendo que a maioria frequentou até ao $12^{\circ}$ ano $(68.2 \%$ homens, $61.5 \%$ mulheres $)$ e os restantes concluíram estudos de nível superior. A maioria dos participantes trabalhava por conta de outrem $(73 \%$ mulheres, 70\% homens), o rendimento anual do casal mais comummente reportado estava entre os $18.375 €$ e os $42.259 €$ (45.2\%), e $12.1 \%$ dos participantes encontravam-se desempregados (5.9\% mulheres; $6.2 \%$ homens).

\section{Instrumentos}

Percepção de pressão económica

A percepção de pressão económica foi avaliada através de quatro indicadores, originalmente desenvolvidos por Conger e colaboradores $(1992 ; 1999)$ e adaptados pelas autoras para o presente estudo. 0 primeiro indicador Dificuldade em pagar contas foi avaliado através do item "Em que medida a 
sua família tem dificuldade em pagar as contas mensais?", com uma escala de Likert de 1 (Não temos dificuldade nenhuma) a 5 (Temos mesmo muitas dificuldades). 0 indicador Dificuldade em poupar dinheiro, foi avaliado através da questão "Em que medida a sua família consegue poupar dinheiro por mês?", com uma escala de Likert de 1 (Não consegue poupar dinheiro) a 4 (Consegue poupar bastante dinheiro). Os casais respondiam ainda a cinco questões, numa escala de Likert de 1 (Discordo totalmente) a 5 (Concordo totalmente), que avaliavam as Preocupações financeiras (e.g., "Não sei como serei capaz de me sustentar nos próximos 12 meses"). 0 quarto indicador Ajustamentos financeiros incluía 28 itens dicotómicos ( $\operatorname{sim} /$ não) acerca de cortes nas despesas que o casal teve necessidade de fazer durante $o$ último ano, devido às dificuldades financeiras (e.g., "Adiei cuidados de saúde médicos/dentários"). Pontuações mais elevadas correspondem a níveis mais elevados de dificuldades em pagar contas e efectuar poupanças, bem como de preocupações e ajustamentos financeiros. Os resultados dos homens e das mulheres foram combinados através do cálculo da média do casal, para cada um dos indicadores. Os níveis de consistência interna foram adequados: Dificuldade em pagar contas $\alpha=.87$; Dificuldade em poupar dinheiro $\alpha=.85$; Preocupações Financeiras: $\alpha=.91$; Ajustamentos Financeiros: $\alpha=.95$.

\section{Stress emocional}

O stress emocional dos cônjuges foi avaliado através do Inventário de Sintomas Psicopatológicos (BSI; Derogatis, 1982; versão portuguesa de Canavarro, 1999), que avalia sintomas psicopatológicos numa escala de Likert de 1 (Nunca) a 5 (Muitíssimas vezes). Apenas três escalas foram utilizadas: Depressão (6 itens; e.g., Sentir que não tem valor), Ansiedade (6 itens; e.g., Nervosismo ou tensão interior) e Hostilidade (5 itens; e.g., Entrar facilmente em discussão). Todas as escalas revelaram um nível de consistência interna adequado para homens e mulheres, respectivamente: Depressão $(\alpha=.85, \alpha=.88)$; Ansiedade $(\alpha=.83, \alpha=$ .86); e Hostilidade ( $\alpha=.83, \alpha=.85)$. Devido à elevada correlação entre os resultados de ansiedade e depressão ( $r=.82$ para as mulheres e $r=.79$ para os homens), as respostas foram agregadas num compósito de Ansiedade-Depressão. Pontuações elevadas reflectem níveis elevados de sintomatologia depressiva/ansiosa e de hostilidade.

\section{Conflito conjugal}

O conflito conjugal foi avaliado através da O'Leary-Porter Scale (OPS; Porter \& O'Leary, 1980; versão portuguesa de Pedro \& Francisco, 2014). A OPS avalia a percepção dos pais sobre a frequência com que o conflito conjugal ocorre na presença dos filhos. A escala inclui 10 itens acerca da frequência com que ocorre agressão verbal e física (e.g., Que percentagens de discussões entre si e o/a seu/sua companheiro/a diria que acontecem em frente a este/a filho/a?) e com que o casal discute sobre a disciplina e hábitos do cônjuge (e.g., Com que frequência é que reclama com o/a seu/sua companheiro/a acerca dos vícios pessoais dele/dela em frente a este(a) filho(a)?). As questões são respondidas com base numa escala de Likert de 1 (Nunca) a 5 (Muito frequentemente). Pontuações mais elevadas correspondem a um maior nível de conflito interparental. A OPS revelou níveis adequados de consistência interna para homens $(\alpha=.78)$ e mulheres $(\alpha=.79)$.

\section{Satisfação conjugal}

A satisfação conjugal foi avaliada através da Kansas Marital Satisfaction Scale (KMSS; Schumm et al., 1986; versão portuguesa de Antunes, Francisco, Pedro, Ribeiro, \& Santos, 2014). A KMSS é uma escala breve constituída por três itens relativos à satisfação com o cônjuge, com o casamento e com a relação de casal (e.g., Em que medida está satisfeito(a) com a sua relação de casal?) numa escala de Likert de 1 (Extremamente insatisfeito/a) a 7 (Extremamente Satisfeito/a). A escala apresenta níveis elevados de consistência interna para homens e mulheres $(\alpha=.98)$. Resultados elevados reflectem níveis elevados de satisfação conjugal.

\section{Procedimento}

Os participantes foram recrutados para uma investigação mais abrangente, acerca das relações familiares em contexto de crise económica, de duas formas: (1) através de oito escolas públicas do $3^{\circ}$ ciclo e do ensino secundário da zona da Grande Lisboa; e (2) através do método "bola de neve", por intermédio de contactos individuais com as famílias. 0 estudo foi aprovado pela Comissão de Deontologia da Universidade a que as autoras pertencem e pelo Ministério da Educação de Portugal. Depois da autorização do Conselho Executivo de cada escola, foi solicitada a colaboração dos casais através da distribuição de cartas aos respectivos filhos, alunos do $7^{\circ}$ ao $12^{\circ}$ ano de escolaridade. Todos os pais entregaram o consentimento informado e os questionários foram enviados para casa através dos filhos, num envelope selado. Cada envelope continha dois conjuntos de questionários (um para o pai e outro para a mãe), com instruções para que respondessem em separado e de forma independente. De modo a 
clarificar dúvidas que pudessem surgir por parte dos pais, foi fornecido o contacto do investigador principal. Os questionários foram entregues pelos pais aos professores responsáveis das turmas participantes, ou aos investigadores responsáveis.

\section{Análises Estatísticas}

Numa primeira fase, foi realizada a análise descritiva dos dados e analisadas as correlações entre as variáveis, através do software SPSS Statistics 22. De seguida, o modelo de mediação foi testado através da Análise de Equações Estruturais (Structural Equation Modeling - SEM), usando o método da máxima verosimilhança com recurso ao software AMOS 22 (Arbuckle, 2012). Para o tratamento dos valores omissos, foi aplicado o método de imputação do algoritmo Expectation Maximization (EM), através do SPSS. O método EM é um procedimento iterativo que consiste em encontrar estimativas de máxima verosimilhança, no qual os parâmetros são estimados através de dois passos: (1) Expectation - os parâmetros são estimados a partir de métodos de regressão com base nos dados observados; (2) Maximization - são calculados novos valores para os parâmetros com base nos valores imputados para os dados omissos e nos dados originais observados. Os dois passos são repetidos até que haja convergência entre as estimativas. Este método produz estimativas menos enviesadas comparativamente a outros procedimentos de tratamento de dados omissos (e.g., substituição de médias) (Schlomer, Bauman, \& Card, 2010). De acordo com Hu e Bentler (1999), a avaliação do ajustamento do modelo aos dados foi realizada com base na análise dos seguintes índices de ajustamento: qui-quadrado $\left(\chi^{2}\right)$, comparative fit index (CFI), root-mean-square error of approximation (RMSEA), e standardized root-mean-square residual (SRMR). Segundo os autores, valores de CFI $>.95$, de RMSEA $<.06$ e de SRMR $<.08$, são indicativos de um bom ajustamento do modelo aos dados. Posteriormente, foi utilizado o método de reamostragem bootstrap (Shrout \& Bolger, 2002) para testar a significância dos efeitos indirectos.

\section{RESULTADOS}

\section{Estatística Descritiva e Análise de Correlações}

A estatística descritiva e as correlações entre as variáveis são apresentadas no Quadro 1. No geral, as correlações são consistentes com o padrão de relações esperado. A percepção de pressão económica apresenta-se positivamente correlacionada com o stress emocional e com o conflito conjugal em ambos os cônjuges, e negativamente correlacionada com a satisfação conjugal dos homens. Relativamente ao stress emocional, observam-se correlações positivas com o conflito conjugal e correlações negativas com a satisfação conjugal. 0 stress emocional dos homens encontra-se ainda positivamente correlacionado com o conflito reportado pelas mulheres e negativamente correlacionado com a satisfação conjugal das mulheres. 0 stress emocional das mulheres encontra-se positivamente correlacionado com o conflito reportado pelos homens e negativamente com a satisfação conjugal dos homens.

\section{o Papel Mediador do Stress Emocional}

Os índices de ajustamento revelam que o modelo conceptual proposto é adequado aos dados: $\chi 2$ (113, $N=$ $208)=206.07, p<.001, \mathrm{CFI}=.97, \mathrm{RMSEA}=.06, \mathrm{SRMR}=.076$, SRMR $=.076$. Todos os itens apresentam um peso factorial superior a .40. Procedeu-se ainda à análise dos índices de modificação (IM), considerandose que parâmetros com IM superiores a 11 eram indicadores de melhoria no ajustamento do modelo (Maroco, 2010). Os valores dos IM não sugeriram modificações no modelo proposto.

Os resultados indicam a presença de efeitos directos entre a pressão económica percepcionada pelo casal e o conflito conjugal, tanto para homens $(\beta=.22, p<.05)$ como para mulheres $(\beta=.25, p<.01)$. De igual modo, foram também encontrados efeitos directos entre a percepção de pressão económica e a satisfação conjugal dos homens $(\beta=-.19, p<.05)$, mas não entre a percepção de pressão económica e a satisfação conjugal das mulheres $(\beta=-.11, p>.10)$. Verificaram-se ainda efeitos directos entre a percepção de pressão económica e o stress emocional dos homens $(\beta=.44, p<.001)$ e das mulheres $(\beta=$ $.55, p<.001)$. Quanto aos actor effects, foram observados efeitos directos entre o stress emocional e o conflito e satisfação conjugal, para homens (conflito $\beta=.58, p<.001$; satisfação $\beta=-.43, p<.001$ ) e para mulheres (conflito $\beta=.31, p<.01$; satisfação $\beta=-.23 p<0.5$ ). Apenas se verificaram partner effects entre o stress emocional dos homens e o conflito e a satisfação conjugal das mulheres $(\beta=.24, p<.01 ; \beta=-.21, p<$ .05 , respectivamente). Não foram observados partner effects entre o stress emocional das mulheres e 0 conflito e satisfação conjugal dos homens $(\beta=.09, p>.05 ; \beta=-.08, p>.05)$.

Relativamente aos efeitos indirectos, os resultados indicam que o stress emocional dos homens e das mulheres medeia a relação entre a percepção de pressão económica e a satisfação conjugal. Nomeadamente, verificaram-se efeitos indirectos entre a percepção de pressão económica e o conflito conjugal dos homens $(\beta=.31, p<.001)$, conflito conjugal das mulheres $(\beta=.27, p<.001)$, satisfação conjugal dos homens $(\beta=-.29, p<.001)$ e satisfação conjugal das mulheres $(\beta=-.22, p<.001)$ (Figura 1). 
Quadro 1. Intercorrelações entre Percepção de Pressão Económica, Stress Emocional, Conflito Conjugal e Satisfação Conjugal (N = 416).

\begin{tabular}{|c|c|c|c|c|c|c|c|c|c|c|c|c|c|c|c|c|c|c|}
\hline Variável & 1 & 2 & 3 & 4 & 5 & 6 & 7 & 8 & 9 & 10 & 11 & 12 & 13 & 14 & 15 & 16 & 17 & 18 \\
\hline \multicolumn{19}{|c|}{ Percepção de Pressão Económica } \\
\hline 1. Poupança C & - & & & & & & & & & & & & & & & & & \\
\hline 2. Cortes C & $.23^{* *}$ & - & & & & & & & & & & & & & & & & \\
\hline 3. Preoc. C & $.56^{* *}$ & $.43^{* *}$ & - & & & & & & & & & & & & & & & \\
\hline 4. Dif. Pag. Cont. C & $.62^{* *}$ & $.46^{* *}$ & $.75^{* *}$ & - & & & & & & & & & & & & & & \\
\hline \multicolumn{19}{|l|}{ Stress Emocional } \\
\hline 5.Hostilidade M & .07 & $.15^{*}$ & $.38^{* *}$ & $.24^{* *}$ & - & & & & & & & & & & & & & \\
\hline 6. AnsDep M & $.20^{* *}$ & $.22^{* *}$ & $.54^{* *}$ & $.39^{* *}$ & $.80^{* *}$ & - & & & & & & & & & & & & \\
\hline 7. Hostilidade $\mathrm{H}$ & .13 & $.25^{* *}$ & $.35^{* *}$ & $.27^{* *}$ & $.33^{* *}$ & $.27^{* *}$ & - & & & & & & & & & & & \\
\hline 8. AnsDep H & $.14^{*}$ & $.25^{* *}$ & $.39^{* *}$ & $.25^{* *}$ & $.232^{* *}$ & $.25^{* *}$ & $.75^{* *}$ & - & & & & & & & & & & \\
\hline \multicolumn{19}{|l|}{ Conflito Conjugal } \\
\hline 9. Conflito M P1 & .01 & $.21^{* *}$ & $.16^{*}$ & $.17^{*}$ & $.28^{* *}$ & $.27^{* *}$ & $.23^{* *}$ & $.20^{* *}$ & - & & & & & & & & & \\
\hline 10. Conflito M P2 & .04 & $.13^{*}$ & .02 & .01 & $.31^{* *}$ & $.22^{* *}$ & $.17^{*}$ & $.17^{*}$ & $.65^{* *}$ & - & & & & & & & & \\
\hline 11. Conflito H P1 & .00 & $.17^{*}$ & $.15^{*}$ & .13 & $.25^{* *}$ & $.14^{*}$ & $.32 *$ & $.35^{* *}$ & $.49^{* *}$ & $.47^{* *}$ & - & & & & & & & \\
\hline 12. Conflito H P2 & .07 & $.17^{*}$ & .07 & .06 & $.18^{* *}$ & .12 & $.39^{* *}$ & $.41^{* *}$ & $.36^{* *}$ & $.55^{* *}$ & $.70^{* *}$ & - & & & & & & \\
\hline \multicolumn{19}{|l|}{ Satisfação Conjugal } \\
\hline 13. Satisfação M P1 & -.02 & -.06 & -.10 & -.09 & $-.20^{* *}$ & $-.19^{* *}$ & -.13 & $-.21^{* *}$ & $-.36^{* *}$ & $-.60^{* *}$ & $-.25^{* *}$ & $-.37 * *$ & - & & & & & \\
\hline 14. Satisfação M P2 & -.01 & -.10 & -.10 & -.05 & $-.25^{* *}$ & $-.22^{* *}$ & $-.14^{*}$ & $-.22^{* *}$ & $-.34^{* *}$ & $-.60^{* *}$ & $-.25^{* *}$ & $-.35^{* *}$ & $.92^{* *}$ & - & & & & \\
\hline 15. Satisfação M P3 & -.03 & -.05 & -.06 & -.07 & $-.19^{* *}$ & $-.18^{* *}$ & -.11 & $-.18^{* *}$ & $-.32^{* *}$ & $-.58^{* *}$ & $-.24^{* *}$ & $-.35^{* *}$ & $.96^{* *}$ & $.93^{* *}$ & - & & & \\
\hline 16. Satisfação H P1 & -.05 & $-.13^{*}$ & $-.11^{*}$ & -.08 & $-.21^{* *}$ & $-.19^{* *}$ & $-.30^{* *}$ & $-.35^{* *}$ & $-.25^{* *}$ & $-.42^{* *}$ & $-.38^{* *}$ & $-.50^{* *}$ & $.54^{* *}$ & $.54^{* *}$ & $.53^{* *}$ & - & & \\
\hline 17. Satisfação H P1 & .08 & $-.11^{*}$ & $-.11 *$ & -.05 & $-.23^{* *}$ & $-.19^{* *}$ & $-.32^{* *}$ & $-.35^{* *}$ & $-.24^{* *}$ & $-.40^{* *}$ & $-.37^{* *}$ & $-.47^{* *}$ & $.53^{* *}$ & $.55^{* *}$ & $.53^{* *}$ & $.93^{* *}$ & - & \\
\hline 18. Satisfação H P3 & .05 & -.09 & -.09 & -.05 & $-.24^{* *}$ & $-.21^{* *}$ & $-.31^{* *}$ & $-.34^{* *}$ & $-.22^{* *}$ & $-.41^{* *}$ & $-.36^{* *}$ & $-49^{* *}$ & $.54^{* *}$ & $.56^{* *}$ & $.55^{* *}$ & $.95^{* *}$ & $.93^{* *}$ & - \\
\hline$M$ & 3.44 & 9.05 & 2.74 & 2.64 & 1.97 & 1.98 & 1.83 & 1.74 & 10.81 & 10.03 & 10.38 & 9.71 & 5.51 & 5.58 & 5.59 & 5.76 & 5.82 & 5.79 \\
\hline$S D$ & .711 & 6.16 & .919 & .933 & .789 & .790 & .672 & .625 & 3.11 & 2.85 & 3.20 & 2.66 & 1.34 & 1.34 & 1.33 & 1.19 & 1.14 & 1.17 \\
\hline
\end{tabular}

Nota. ${ }^{* *} p<.01, * p<.05$

C= Casal; M= Mulher; H= Homem; Preoc. = Preocupações Financeiras; Dif. Pag. Cont. = Dificuldade em Pagar Contas; AnsDep = Ansiedade-Depressão; P1 a P3 = Parcelas 


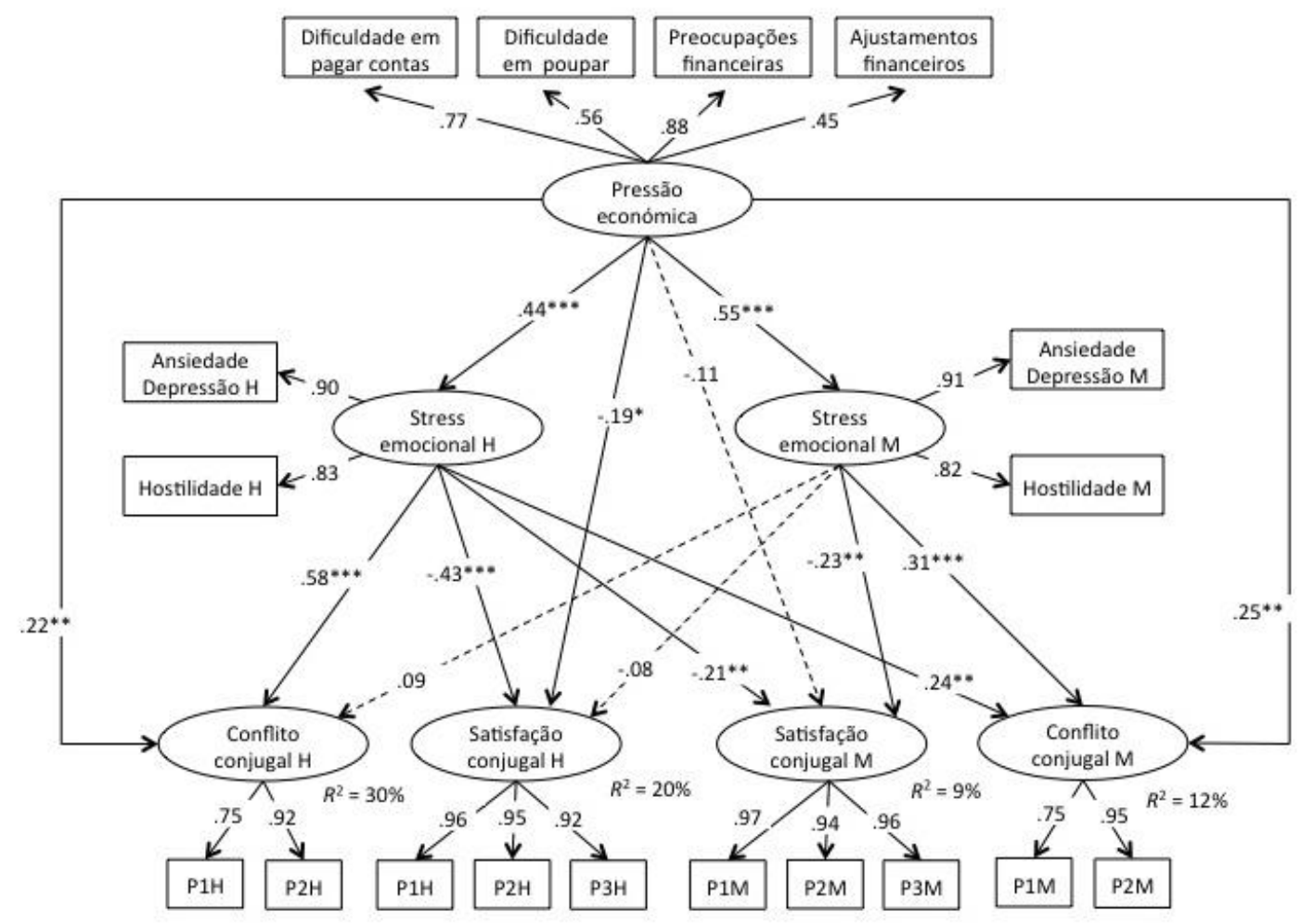

Figura 1. Coeficientes estandardizados do modelo de mediação proposto, da relação entre percepção de pressão económica, stress emocional e conflito e satisfação conjugal.

Nota: $H$ = homens; $M$ = mulheres; $P 1 H-P 3 H$ = parcelas relativas aos homens da O'Leary-Porter Scale e da Escala de Satisfação Conjugal de Kansas; P1M - P3M = parcelas relativas às mulheres da O'Leary-Porter Scale e da Escala de Satisfação Conjugal de Kansas. Os distúrbios das variáveis de stress emocional foram correlacionados entre si, bem como os distúrbios das variáveis de conflito e satisfação conjugal. Por questões de parcimónia, os distúrbios e os resíduos não são apresentados no modelo. ${ }^{*} p<.05 .{ }^{* *} p<.01 .{ }^{* * *} p<$ .001 .

\section{DISCUSSÃO}

O presente estudo pretendeu investigar o papel mediador do stress emocional de ambos os elementos do casal, na relação entre percepção de pressão económica e conflito e satisfação conjugais. Procurou-se ainda alargar a compreensão do modelo de stress familiar económico (Conger \& Elder, 1994), explorando a influência mútua e interdependência entre cônjuges, através de uma análise de dados baseada no ActorPartner Interdependence Model.

Os resultados suportam a primeira hipótese, referente à existência de efeitos directos entre a percepção de pressão económica e o conflito conjugal, na linha do que havia sido encontrado em estudos anteriores realizados na Coreia (Kwon et al., 2003), Turquia (Aytaç \& Rankin, 2009), Finlândia (Kinnunen \& Feldt, 2004) e Bélgica (Ponet et al., 2013). Os dados apoiam assim a ideia de que, apesar da maioria dos estudos realizados nos EUA demonstrar um impacto indirecto das dificuldades financeiras no funcionamento conjugal, em países fora dos EUA a percepção de pressão económica parece exercer uma influência tanto directa como indirecta na qualidade conjugal (Conger et al., 2010). Quando as sociedades experienciam choques económicos severos, estes exercem um impacto mais forte nas finanças familiares e nos seus contextos sociais, existindo uma maior tendência para uma relação directa entre a percepção de dificuldades económicas e a qualidade da relação conjugal (Kwon et al., 2003). Os dados deste estudo podem ser interpretados neste sentido, considerando que a população portuguesa tem vindo a sofrer cortes constantes nas suas finanças e nas medidas de apoio social por parte do Governo, ao mesmo tempo que o custo da qualidade de vida tem vindo a aumentar (INE, 2013). A existência de diferenças culturais entre famílias latinas e anglo-americanas, ao nível do significado atribuído ao dinheiro, poderá também ajudar a compreender estes efeitos directos. Segundo Falicov (2001), os latinos valorizam mais o tempo de lazer familiar, considerando o dinheiro como uma forma de promover as interacções familiares e conjugais, e os bens materiais como algo que desenvolve a coesão e os laços entre os membros da família. O dinheiro é assim utilizado de várias maneiras para a família (e.g., presentes, realização de rituais familiares), estando o consumo relacionado com a qualidade da vida familiar, em geral, e conjugal, em particular. Considerando que a crise financeira em Portugal diminuiu consideravelmente o poder de 
compra das famílias e a possibilidade de promover a coesão e qualidade conjugal através de actividades que impliquem despesas económicas, reduzindo ainda o tempo de lazer dedicado ao casal (eventualmente devido à acumulação de empregos ou à realização de horas extraordinárias no trabalho), estas diferenças culturais poderão ajudar a explicar os efeitos directos observados entre a percepção de pressão económica e a qualidade conjugal.

Importa referir as diferenças encontradas entre homens e mulheres. Em consonância com os resultados de Aniol e Snyder (1997), foram encontrados efeitos directos entre a percepção de pressão económica e a satisfação conjugal masculina, mas não entre a pressão económica e a satisfação conjugal feminina. Para os homens, a satisfação conjugal mostrou-se directamente relacionada com a percepção de pressão económica vivenciada pelo casal, ao passo que, para as mulheres, a percepção de pressão económica parece relacionar-se apenas indirectamente com a satisfação conjugal feminina, através do aumento do stress emocional. Uma possível explicação prende-se com os papéis de género predominantes na sociedade portuguesa. Portugal viveu num regime ditatorial até à década de 70 , onde, segundo as ideologias do Estado Novo, se vivia numa sociedade fortemente marcada pela diferenciação de género a nível social e familiar: os homens tinham o papel de "chefe de família", sendo considerados responsáveis pelo bem-estar financeiro e recursos económicos, enquanto a mulher era responsável pelo trabalho doméstico e bem-estar emocional dos filhos (Wall, Aboim, \& Cunha, 2010). Por outro lado, nas famílias latinas ainda se observa uma tendência de controlo do dinheiro por parte do homem (Falicov, 2001), sendo o ordenado da mulher percepcionado como secundário (Strandh et al., 2013). Apesar da sociedade portuguesa estar a caminhar para papéis de género mais igualitários, estas questões culturais ainda persistem na actualidade. Neste sentido, alguns estudos demonstram que a relação entre dificuldades económicas e ajustamento conjugal é mais pronunciada para os homens, e que o comportamento e stress masculinos estão mais fortemente associados a problemas financeiros (Conger et al., 1990; Liker \& Elder, 1983). Para os homens, mais do que para as mulheres, o dinheiro está intimamente associado a aspectos do funcionamento do casal, como questões de poder na relação conjugal, bem como com sentimentos de valor pessoal e auto-estima (Papp et al., 2009). Assim, a percepção de pressão económica poderá originar sentimentos de auto-depreciação e baixa auto-estima nos homens, afectando a sua satisfação conjugal. Estes resultados parecem ainda evidenciar que os homens têm maior tendência para experienciar spillover de stress de um domínio da vida (dificuldades financeiras) para outro domínio (relação conjugal), ao passo que as mulheres apresentam maior facilidade em compartimentalizar o stress e evitar o spillover de tensão entre diferentes domínios, como constatado em estudos anteriores (Almeida, Wethington, \& Chandler, 1999; Larson \& Almeida, 1999).

No que diz respeito à segunda hipótese, os dados confirmaram a presença de partner effects para os homens, mas não para as mulheres. Nomeadamente, foram encontrados efeitos directos entre o stress emocional masculino e o conflito e a satisfação conjugal das mulheres, mas não entre o stress emocional feminino e o conflito e satisfação conjugal dos homens. Para as mulheres, apenas foram observados actor effects. Estes resultados são congruentes com estudos que sugerem que o crossover entre cônjuges tende a ser unidirecional, dos homens para as mulheres (Bolger et al., 1989; Jones \& Fletcher, 1993; Larson \& Almeida, 1999; Pittman et al., 1996). Uma possível explicação para este dado poderá estar relacionada com evidências recentes que mostram que quando os casais experienciam dificuldades financeiras, os homens tendem a afastar-se do cônjuge, dado o seu desejo de mais independência relacional, enquanto as mulheres revelam maior necessidade de proximidade na relação (Falconier \& Epstein, 2011), focando mais a sua atenção no comportamento e investimento relacional do companheiro, um aspecto crítico para a sua própria satisfação (Bodenmann, Pihet, \& Kayser, 2006). Considerando os papéis de género tradicionais que ainda caracterizam a sociedade portuguesa, os elementos do casal podem assumir que é da responsabilidade do homem resolver os problemas financeiros. As mulheres podem tender a abordar os maridos para procurar soluções, considerando os homens que a resolução dos problemas financeiros é da sua responsabilidade, afastando-se da companheira na tentativa de resolver os problemas sozinho (Falconier \& Epstein, 2011). Se o homem é percepcionado como distante e desinteressado, os níveis de satisfação femininos diminuem. Estes resultados contribuem para a literatura que demonstra diferenças de género no MSFE (e.g., Falconier \& Epstein, 2011; Kwon et al., 2003; Ponnet et al., 2013).

Por fim, relativamente à terceira hipótese, os resultados são consistentes com os estudos que demonstram uma relação indirecta entre a percepção de pressão económica e o conflito e satisfação conjugais, mediada pelos níveis de stress emocional dos cônjuges (e.g., Conger et al., 2002; Cutrona et al., 2003; Kinnunen \& Feldt, 2004). Quando os casais experienciam maior pressão económica, esta parece estar associada a sintomatologia depressiva e ansiosa, bem como a comportamentos de maior hostilidade entre os elementos do casal, aumentando o conflito entre os cônjuges e diminuindo a satisfação conjugal. Os dados sugerem, assim, a aplicabilidade do modelo de stress familiar económico ao contexto português. 
É ainda de salientar que os dados revelam a importância de se investigar as repercussões das dificuldades financeiras em famílias de nível socioeconómico médio, e não apenas em famílias em risco de pobreza. Em casais que não experienciam dificuldades económicas objectivas severas, mas que sofreram cortes nos rendimentos e apresentam preocupações financeiras, por exemplo, a percepção de pressão económica exerce ainda assim um impacto considerável a nível individual e conjugal. Mais do que avaliar indicadores financeiros objectivos, é fundamental analisar a vivência subjectiva da pessoa, relativamente à percepção de pressão económica.

\section{Limitações e Implicações Clínicas}

Apesar do presente estudo contribuir para uma melhor compreensão do modelo de stress familiar económico, devem ser referidas algumas limitações, como a natureza transversal do estudo que impossibilita a identificação de causalidade entre as variáveis. Por outro lado, o facto de a amostra não reunir um maior número de famílias de estatuto socioeconómico baixo poderá ter afectado os resultados, diminuindo o poder de generalização dos mesmos. Por último, o procedimento de aplicação dos protocolos, no qual os casais responderam aos questionários em casa, sem a presença dos investigadores, não permitiu assegurar a resposta às questões de forma individual e confidencial. Apesar destas limitações, o estudo apresenta importantes implicações para a prática clínica. Em sociedades com papéis de género mais tradicionais, onde os homens são considerados os principais responsáveis pelo sustento da família, os terapeutas deverão estar particularmente atentos à pressão económica percepcionada pelos homens. Os resultados sugerem que, em contexto de dificuldades financeiras, e mesmo em famílias de nível socioeconómico médio, a intervenção clínica deverá centrar-se em fornecer recursos aos homens que lhes permitam diminuir o spillover de stress provocado pela percepção de pressão económica, atenuando o impacto no conflito e satisfação conjugal masculina, e prevenindo a diminuição da satisfação conjugal feminina.

Em conclusão, este estudo constituiu a primeira aplicação do modelo de stress familiar económico ao contexto português, comprovando a influência directa e indirecta da percepção de pressão económica ao nível do conflito e satisfação conjugais. Os dados permitiram ainda uma melhor compreensão dos efeitos de género subjacentes e da complexidade das interinfluências entre cônjuges, em situação de crise económica.

\section{Referências}

Aboim, S. (2010a). Family and gender values in contemporary Europe: The attitudinal gender gap from a crossnational perspective. Portuguese Journal of Social Science, 9, 33-58.

Aboim, S. (2010b). Gender cultures and the division of labour in contemporary Europe: A cross-national perspective. The Sociological Review, 58, 171-196.

Almeida, D., Wethington, E., \& Chandler, A. (1999). Daily transmission of tensions between marital dyads and parent-child dyads. Journal of Marriage and the Family, 61, 49-61.

Amâncio, L. (2007). Género e divisão do trabalho doméstico - 0 caso português em perspectiva. In K. Wall \& L. Amâncio (Eds.), Família e género em Portugal e na Europa (pp. 181-209). Lisboa: ICS.

Aniol, J., \& Snyder, D. (1997). Differential assessment of financial and relationship distress: Implications for couples therapy. Journal of Marital and Family Therapy, 23, 347-352.

Antunes, N., Francisco, R., Pedro, M., Ribeiro, M.T., \& Santos, S. (2014). Escala de satisfação conjugal de Kansas [versão para investigação]. Lisboa: Universidade de Lisboa.

Arbuckle, J. L. (2012). Amos 21.0 User's guide. Chicago: SPSS, IBM.

Aytaç. I., \& Rankin, B. (2009). Economic crisis and marital problems in Turkey: Testing the family model stress. Journal of Marriage and Family, 71, 756-767.

Bodenmann, G., Pihet, S., \& Kayser, K. (2006). The relationship between dyadic coping and marital quality: A 2 year longitudinal study. Journal of Family Psychology, 20, 485-493.

Bolger, N., DeLongis, A., Kessler, R. C., \& Wethington, E. (1989). The contagion of stress across multiple roles. Journal of Marriage and the Family, 51, 175-183.

Brinkman, H. J., Pee, S., Sanogo, I., Subran, L., \& Bloem, M. (2010). High food prices and the global financial crisis have reduced access to nutritious food and worsened nutritional status and health. The Journal of Nutrition, 140, 153S-161S.

Canavarro, M. C. (1995). Inventário de Sintomas Psicopatológicos. In M. Simões, M. Gonçalves, \& L. Almeida (Eds.), Testes e Provas Psicológicas em Portugal - Vol. II. (pp. 95-109). Braga: Sistemas Humanos e Organizacionais, Lda.

Conger, R. D., \& Donnellan, M. B. (2007). An interactionist perspective on the socioeconomic context of human development. Annual Review of Psychology, 58, 175-199. 
Conger, R. D., \& Elder, G. H. (1994). Families in troubled times: Adapting to change in rural America. Hawthorne, NY: Aldine de Gruyter.

Conger, R. D., Conger, K., \& Martin, M. (2010). Socioeconomic status, family processes and individual development. Journal of Marriage and Family, 72, 685-704.

Conger, R. D., Conger, K., Elder, G., Lorenz, F., Simons, R., \& Whitbeck, L. (1992). A family process model of economic hardship and adjustment of early adolescent boys. Child Development, 63, 526-541.

Conger, R. D., Elder, G., Lorenz, F., Conger, K., Simns, R., Whitbeck, S., Huck, S., \& Melby, J. (1990). Linking economic hardship to marital quality and instability. Journal of Marriage and the Family, 52, 643656.

Conger, R. D., Rueter, M., \& Elder Jr, G. (1999). Couple resilience to economic pressure. Journal of Personality and Social Psychology, 76, 54-71.

Conger, R. D., Wallace, L., Sun, Y., Simons, R., McLoyd, V., \& Brody, G. (2002). Economic pressure in African American families: A replication and extension of the family stress model. Developmental Psychology, 28, 179-193.

Cutrona, C., Russell, D., Abraham, W. T., Gardner, K., Melby, J., Bryant, C., \& Conger, R.D. (2003). Neighborhood context and financial strain as predictors of marital interaction and marital quality in African American couples. Journal of the International Association for Relationship Research, 10, 389-409.

Dakin, J., \& Wampler, R. (2008). Money doesn't buy happiness, but it helps: Marital satisfaction, psychological distress, and demographic differences between low- and middle-income clinic couples. The American Journal of Family Therapy, 36, 300-311.

Davis, M., \& Mantler, J. (2004). The consequences of financial stress for individuals, families, and society. Report for the Centre for Research on Stress, Coping, and Well-being [PDF]. Retirado de http://www.researchgate.net/profile/Janet_Mantler/publication/229052873_The_Consequences_ of_Financial_Stress_for_Individuals_Families_and_Society/links/0c9605295f3d47acb9000000.pdf

DECO (2013). Travar o sobre-endividamento. Associação Portuguesa para a Defesa do Consumidor. Retirado de http://www.deco.proteste.pt/dinheiro/orcamento-familiar/dossie/travar-sobreendividamento

Dew, J. (2008). Debt change and marital satisfaction change in recently married couples. Interdisciplinary Journal of Applied Family studies, 57, 60-71.

Economou, M., Madianos, M., Peppou, L., \& Patelakis, A. (2012). Major depression in the era of economic crisis: A replication of a cross-sectional study across Greece. Journal of Affective Disorders, 145, 308-314.

Falconier, M., \& Epstein, N. (2011). Female-demand/male-withdraw communication in Argentinian couples: A mediating factor between economic strain and relationship distress. Personal Relationships, 18, 586-603.

Falicov, C. (2001). The cultural meanings of money: The case of Latinos and Anglo-Americans. American Behavioral Scientist, 45, 313-328.

Fincham, F., Stanley, S., \& Beach, S. (2007). Transformative processes in marriage: An analysis of emerging trends. Journal of Marriage and Family, 69, 275-292.

Gudmunson, C. G., Beutler, I.F., Israelsen, C.L., McCoy, J.K., \& Hill, E.J. (2007). Linking financial strain to marital instability: Examining the roles of emotional distress and marital interaction. Journal of Family and Economic Issues, 28, 357-376.

Hu, L. T., \& Bentler, P. (2009). Cutoff criteria for fit indexes in covariance structure analysis: Conventional criteria versus new alternatives. Structural Equation Modeling, 6, 1-55.

INE (2013). População residente em risco de pobreza ou exclusão social. Inquérito às Condições de Vida e Rendimento. Retirado de

http://www.ine.pt/xportal/xmain?xpid=INE\&xpgid=ine_indicadores\&indOcorrCod=0006271\&con texto $=$ bd\&selTab=tab2

Jones, F., \& Fletcher, B. (1993). An empirical study of occupational stress transmission in working couples. Human Relations, 46, 881-903.

Kenny, D., Kashy, D., \& Cook, W. (2006). Dyadic data analysis. New York: The Guilford Press.

Kinnunen, U., \& Feldt, T. (2004). Economic stress and marital adjustment among couples: Analyses at the dyadic level. European Journal of Social Psychology, 34, 519-532.

Kwon, H. K., Rueter, M., Lee, M.S., Koh, S., \& Ok, S. (2003). Marital relationships following the Korean economic crisis: Applying the family stress model. Journal of Marriage and Family, 65, 316-325.

Larson, R., \& Almeida, D. (1999). Emotional transmission in the daily lives of families: A new paradigm for studying family process. Journal of Marriage and the Family, 61, 5-20. 
Liker, J., \& Elder Jr, G. (1983). Economic hardship and marital relations in the 1930s. American Sociological Review, 48, 343-359.

Maroco, J. (2010). Análise de equações estruturais: Fundamentos teóricos, software \& aplicações. Lisboa, Portugal: ReportNumber.

Matud, M. P. (2004). Gender differences in stress and coping styles. Personality and Individual Differences, 37, 1401-1415.

Mistry, R., Lowe, E., Benner, A., \& Chien, N. (2008). Expanding the family economic stress model: Insights from a mixed-methods approach. Journal of Marriage and Family, 70, 196-209.

Neff, L., \& Karney, B. (2007). Stress crossover in newlywed marriage: A longitudinal and dyadic perspective. Journal of Marriage and Family, 69, 594-607.

Papp, L., Cummings, E. M., \& Goeke-Morey, M. (2009). For richer, for poorer: Money as a topic of marital conflict in the home. Family Relations, 58, 91-103.

Pedro, M., \& Francisco, R. (2014). Escala de O'Leary-Porter [versão para investigação]. Lisboa: Universidade de Lisboa.

Pittman, J. E., Solheim, C. A., \& Blanchard, D. (1996). Stress as a driver of the allocation of house work. Journal of Marriage and the Family, 58, 456-468.

Ponnet, K., Wouters, E., Goedemé, T., \& Mortelmans, D. (2013). Family financial stress, parenting and problem behavior in adolescents: An actor-partner interdependence approach. Journal of Family Issues, 20 (10), 1-24.

Porter, B., \& O'Leary, D. (1980). Marital discord and childhood behavior problems. Journal of Abnormal Child Psychology, 8, 287-295.

Robila, M., \& Krishnakumer, A. (2005). Effects of economic pressure on marital conflict in Romania. Journal of Family Psychology, 19, 246-251.

Schlomer, G. L., Bauman, S., \& Card, N. A. (2010). Best practices for missing data management in counseling psychology. Journal of Counseling Psychology, 57, 1-10.

Schumm, W., Paff-Bergen, L., Hatch, R., Obiorah, F., Copeland, J., Meens, L., \& Bugaighis, M. (1986). Concurrent and discriminant validity of the Kansas marital satisfaction. Journal of Marriage and Family, 48, 381-387.

SEDES (2012). 0 impacto da crise no bem estar dos portugueses. Associação para o Desenvolvimento Económico e Social. Retirado de http://www.sedes.pt/documentacao.aspx?args=2,8\&tipo=artigos\&ID=61

Shrout, P., \& Bolger, N. (2002). Mediation in experimental and nonexperimental studies: New procedures and recommendations. Psychological Methods, 7, 422-445.

Strandh, M., Hammarström, A., Nilsson, K., Nordenmark, M., \& Russel, H. (2013). Unemployment, gender and mental health: The role of the gender regime. Sociology of Health \& Illness, 35, 649-665.

Wall, K., Aboim, S., \& Cunha, V. (2010). A vida familiar no masculino: Negociando velhas e novas masculinidades. Lisboa: Comissão para a Igualdade no Trabalho e no Emprego.

Westman, M. (2001). Stress and strain crossover. Human Relations, 54, 717-751.

Westman, M., Etzion, D., \& Danon, E. (2001). Job insecurity and crossover of burnout in married couples. Journal of Organizational Behavior, 22, 467-481.

Wickrama, K., Hwa Kwag, K., Lorenz, F., Conger, R., \& Surjadi, F. (2010). Dynamics of family economic hardship and the progression of health problems of husbands and wives during the middle years: a perspective from rural Mid-West. Journal of Aging and Health, 22, 1132-1157.

Historial do artigo

Recebido $\quad 18 / 09 / 2014$

Aceite $\quad 30 / 03 / 2015$

Publicado $\quad 06 / 2015$

Apoio à publicação: Fundação para a Ciência e a Tecnologia (Ministério da Educação e Ciência, Portugal) - Programa FACC 